\section{P71 Barriers to Attendance in a Father-focused Adolescent Obesity Prevention Program: Padres Preparados, Jóvenes Saludables}

Sayaka Nagao-Sato, MS, RDN, ssato@umn.edu, University of Minnesota, 1334 Eckles Ave, St. Paul, MN, 55108; Silvia Alvarez de Davila, PhD, University of Minnesota, Extension Center for Family Development; Aysegul Baltaci, MS, University of Minnesota, Department of Food Science and Nutrition; Roxana Linares, $M A$, Centro Tyrone Guzman, Minneapolis; Alejandro Peralta Reyes, $M P H, B S$, University of Minnesota, Extension Center for Family Development; Youjie Zhang, PhD, RDN, Department of Child and Adolescent Health and Social Medicine, School of Public Health, Medical College of Soochow University; Ghaffar Ali Hurtado Choque, PhD, University of Maryland, School of Public Health; Marla Reicks, PhD, $R D N$, University of Minnesota, Department of Food Science and Nutrition

Background: Latino family-centered, community-based intervention studies to prevent childhood obesity have primarily involved mothers; therefore, little information about father participation is available. A Latino fatherfocused program to prevent adolescent obesity was conducted with low participation from some fathers.

Objective: The study aim was to determine factors associated with fathers' attendance in an adolescent obesity prevention program.

Study Design, Settings, Participants: Data were from 147 fathers participating in the Padres Preparados, Jóvenes Saludables program, a randomized controlled trial to prevent Latino adolescent (10-14 years) obesity. Eight weekly (2-hour) sessions were conducted in the Minneapolis/St. Paul metropolitan area (2017-2020). Participants were randomly assigned to intervention or waitlist control groups after baseline data collection.

Measurable Outcome/Analysis: The program facilitator recorded father presence or absence at each session. Attendance data were used in cluster analysis to identify attendance patterns. Predictor variables were measured at baseline with surveys and via anthropometric measures, including demographics, socioeconomic status, adolescent weight status, adolescent weight concerns, foodrelated activities with adolescents, family meal frequency, food assistance program participation, family stress, number of children, neighborhood safety, assigned group, site, and session season. Multinomial logistic regression analyses were used to examine associations between attendance patterns and predictor variables after assessing appropriateness for inclusion in regression models.

Results: Three attendance patterns were identified for all fathers: consistently high attendance (52\%), declining attendance $(18 \%)$, and non-attendance (30\%). Compared to fathers with a consistently high attendance pattern, fathers with a non-attendance pattern were more likely to be assigned to the waitlisted control group (OR: 7.9 [95\% CI: 2.6-23.9]; $P<$ 0.01 ) and have lower household income (OR: 2.8 [95\% CI: 1.0-7.8]; $P=0.04)$. No predictors were associated with declining attendance compared to consistently high attendance.

Conclusion: Maintaining contact with control families during waiting periods and providing online sessions to increase flexibility for low-income families could address Latino father non-attendance.

Funding: NIFA.

\section{P72 Comparison of EFNEP Educators' and Low-Income High School Teens' Opinions of a Game-Based Nutrition and Physical Activity Curriculum}

Het Desai-Saha, BS, het@scarletmail.rutgers.edu, Rutgers, The State University of New Jersey, 26 Nicol Ave, 2nd Floor, New Brunswick, NJ, 08901; Graham E. Bastian, RDN, Rutgers, The State University of New Jersey; Debra Keenan, PhD, M.Ed., Rutgers, The State University of New Jersey

Background: The Expanded Food and Nutrition Education Program (EFNEP) has provided limited education to teens. Thus, differences between educators' and teens' perceptions of lessons taught, particularly when a gamification approach is used, are not available.

Objective: To compare EFNEP educators' and lowincome, urban teens' impressions of a classroom-based, nutrition and physical activity (PA) curriculum that utilized a gamification approach.

Study Design, Settings, Participants: The intervention, a 10-week EFNEP curriculum taught between 20182020, and interviews took place at Brimm High School in Camden, NJ. This was a comparative study inquiring the opinions of EFNEP educators' and 9th-grade teen participants' perceptions of the lessons taught. Researchers conducted semi-structured, 1-on-1, face-to-face interviews with the educators and teens (who attended over $80 \%$ of the lessons).

Measurable Outcome/Analysis: Two independent coders performed a thematic analysis of interview transcripts. Findings were discussed until both researchers reached a consensus.

Results: A high level of agreement was evidenced between the teens' $(n=25)$ and EFNEP educators' $(n=9)$ that gamified lessons prompted greater participation and engagement than other types of lessons. Educators presumed that 2 videos (a walk-indoors DVD featuring an adult cast and a sustainability video encouraging reduced red meat consumption and increased plant-based foods consumption) and a food safety lesson based on a cookout, would not be relatable to teens. However, the teens reported favorable knowledge and/or intention to change regarding their PA (75\%) vegetable intake $(40 \%)$, and food safety practices (>33\%). The curriculum resulted in positive overall impacts regarding the teens' PA (through the use of dance as PA), improved fast food choices, decreased intake of sugar-sweetened beverages, and improved snack choices.

Conclusion: Educators and teens valued gamified lessons as an effective educational approach for working with this 
P72 (continued)

age group; yet with regards to some lesson contents, educators appeared to be far more sensitive to potential issues than did the teens.

Funding: NJ EFNEP.

\section{P73 Cultivating Connections with Farm to School Activities During School Closures}

Patty Case, MS, RD, patty.case@oregonstate.edu, Oregon State University Extension Service, 6923 Washburn Way, Klamath Falls, OR, 97601; Cheryl Kirk, BS, RD, Oregon State University Extension Service Josephine Count

Objective: To engage youth and their families in a webbased hybrid club for farm to school activities as an alternative to school-based activities when schools closed due to the pandemic.

Use of Theory or Research: Farm to school programs increase children's access to and knowledge of fresh and local foods primarily through experiential learning (Kolb's Experiential Learning Theory) such as gardening, tasting food and field trips.

Target Audience: Youth, grades 2-6, and their families who experienced disruption in usual learning and social activities due to COVID-19 restrictions.

Program Description: Experiential activities are the hallmark of farm to school education. In March 2020 all hands-on, school based activities planned by Extension faculty were cancelled due to the pandemic. Faculty responded by offering garden-cooking clubs using a web-based hybrid approach independent of schools. Faculty facilitated biweekly virtual meetings of 30 minutes for 6-8 weeks for youth and their families. Asynchronous activities were also offered via a website, kits, video demonstrations, farmers market vouchers and when possible, farm tours.

Evaluation Methods: Adult caregivers received a survey at the end of the 6-8 week club sessions to evaluate their child's and family's level of engagement including queries on how much time they spent using web-based tools and how likely they were to start a garden.

Results: One hundred and ninety youth, grades $2-6$, and their families participated in 4 virtual and/or hybrid clubs delivered by Extension faculty from March 2020 to October 2020. Caregiver responses from the survey $(n=46)$ indicated $83 \%$ of families expanded or started a garden. On average youth and/or families spent 47 minutes per week engaged in program activities including online learning.

Conclusion: It appears that virtual programming can actively engage youth and their families in farm to school education and may be a tool to supplement resource intensive hands-on strategies in the future. Further research is need to determine which components are best suited for web-based delivery vs in person delivery.

Funding: Oregon Department of Education.
P74 Diet Behavior During CovID: A Qualitative Study of Former Participants of a Food Security Intervention

Miranda Cook, MPH, macook2@emory.edu, Laney Graduate School, Emory University, 1518 Clifton Rd, Atlanta, GA, 30322; David Denton, BS, Rollins School of Public Health, Emory University; Stacie Schmidt, MD, Emory University School of Medicine; Amy Webb Girard, $P h D$, Hubert Department of Global Health, Emory University

Background: The Georgia Food for Health program $(\mathrm{GF} 4 \mathrm{H})$ is a clinic-based nutrition education and food security intervention program that has demonstrated the ability to improve dietary behaviors. However, little is known about changes in behaviors during COVID-related lockdowns.

Objective: To characterize diet behavior changes experienced by former participants of a food security intervention during COVID-related lockdowns.

Study Design \& Setting: We conducted semi-structured interviews by phone with 17 patient participants in May June 2020 with program participants who had completed the program within the past 6 months. Participants were asked questions about the program, diet and stress-related behaviors, and changes in these behaviors within the COVID lockdown period.

Participants: Participants were mainly middle-aged (mean age 59 years), African American (100\%), women (59\%). Approximately half screened positive for food insecurity $(47.1 \%)$. All participants were healthy at the time of the interview.

Analysis: Transcripts of interviews were analyzed for both apriori and emergent themes using thematic analysis.

Results: We identified 3 central themes. First, participants discussed changes in shopping behaviors to minimize time spent in stores using approaches like shopping lists to plan routes within the store or sending younger relatives to shop for them. Due to supply issues at stores, participants discussed adapting recipes to include available options. Second, participants discussed disinterest in cooking, a lack of motivation to engage in healthy eating strategies they had previously adopted, and anticipation of regretting these behaviors post-COVID. Third, participants were largely uncomfortable at the prospect of resuming in-person programming and preferred virtual options due to safety concerns.

Conclusion: Among former participants of the GF4H program, shopping and cooking behaviors shifted during the COVID-19 lockdown period to adapt to safety guidelines and to overcome supply issues. Motivation to engage in healthy cooking and eating behaviors was diminished. Ongoing program efforts should focus on virtual options to engage and re-engage participants.

Funding: $\mathrm{NIH}$. 